\title{
HHG probing of atomic dipoles by electronic wave-packet caustics
}

\author{
Davide Faccialà ${ }^{1,}$, Stefan Pabst $^{2,3}$, Barry D. Bruner ${ }^{4}$, Anna G. Ciriolo ${ }^{1}$, Michele Devetta ${ }^{1}$, \\ Matteo Negro ${ }^{1}$, Hadas Soifer ${ }^{4}$, Nirit Dudovich ${ }^{4}$, Salvatore Stagira ${ }^{5}$, and Caterina Vozzi ${ }^{1}$ \\ ${ }^{1}$ Istituto di Fotonica e Nanotecnologie, CNR, Milan, Italy \\ ${ }^{2}$ Stanford PULSE Institute, SLAC National Accelerator Laboratory, Menlo Park (CA), USA \\ ${ }^{3}$ ITAMP Harvard-Smithsonian Center for Astrophysics, Cambridge (MA), USA \\ ${ }^{4}$ Department of Physics of Complex Systems, Weizmann Institute of Science, Rehovot, Israel \\ ${ }^{5}$ Dipartimento di Fisica, Politecnico di Milano, Milan, Italy
}

\begin{abstract}
We exploit high-order harmonic generation spectroscopy at the caustics of the recombining electron wave-packet as a method for directly comparing experimental spectra with ab-initio theories. Experimental results in xenon and comparison with ab-initio time-dependent configuration-interaction singles calculations allowed to assess the role of the wave-packet enhancement during the giant resonance. Results in argon show that this technique can also be applied to other targets.
\end{abstract}

\section{Introduction}

Direct comparison of high-order harmonic generation (HHG) spectra with ab-initio singleatom theories is often hampered by many factors, such as spatial phase-matching and averaging effects which often reshape the experimental spectrum in a hardly predictable way. Taking into account these effects often requires time-consuming calculations and full knowledge of the experimental parameters. Especially when multi-electronic effects are included, ab-initio calculations in multi-atomic systems are simply not affordable. It is therefore desirable to tailor a probe that can better mimic the single atom response even under a variety of experimental conditions.

Caustics in HHG [1] are shown to possibly represent a good candidate for such a purpose. When the HHG process is driven by a field with a strong component of wavelength $\lambda$ and a weaker second harmonic, two caustics appear in the HHG spectrum. Their presence is due to the coalescence of long and short trajectories at the cutoffs of the two electronic wave-packets recombining from opposite sides of the atom. Thus, by probing the signal at these two spectrally separated caustics, it is possible to isolate and enhance a single recombination event for each cycle of the laser field, overcoming the problem of determining which trajectory is actually contributing to the process. This trajectory selection occurs at the "single-atom level", and it is not due to phase-matching in the target, which suggests a more reliable comparison with single-atom theoretical results.

* Corresponding author: davide.facciala@polimi.it 
In addition, by tuning the relative phase $\varphi$ between the two colors, the energy of the two caustics can be moved over a wide range of values. In this way, we can probe the target at different energies [2].

A careful analysis of the results presented in our previous paper [2] and a comparison with ab-initio and quantitative rescattering (QRS) theories allows to assess the role of the electronic wave-packet enhancement in the xenon giant resonance. In xenon, probing the caustic is indeed facilitated by the presence of the giant-resonance itself, which enhances the HHG signal in the region of interest. This resonance involves both the outermost $5 p$ orbital and the inner $5 \mathrm{~s}$ and $4 \mathrm{~d}$ orbitals [3]. Recent results in argon demonstrate the possibility to extend this technique even to systems that do not show resonances.

\section{Results}

We probed the giant-resonance in xenon with a two color field made by a strong component at wavelength $\lambda=1550 \mathrm{~nm}$ and a weaker second harmonic [2]. The acquired spectra as a function of $\varphi$ are reported in Fig. 1(a).
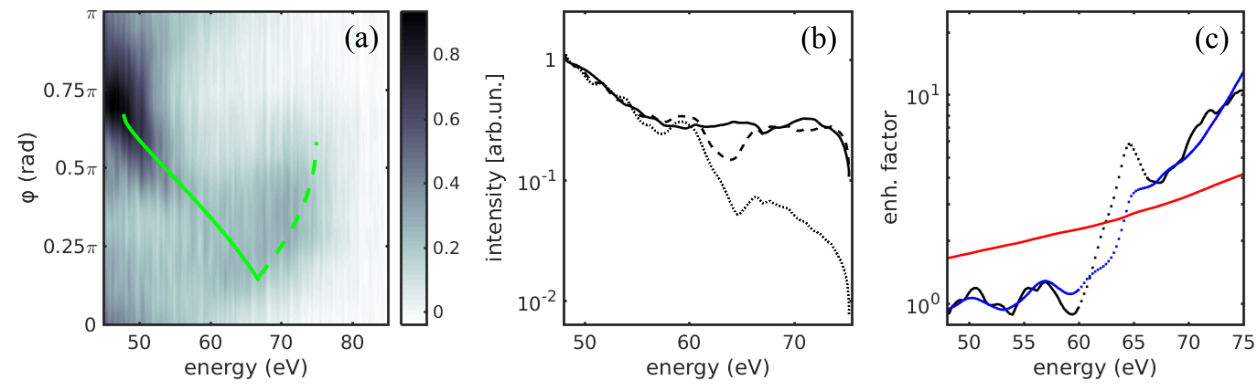

Fig 1. (a) HHG spectra in Xe as a function of $\varphi$, green line indicates the position of the upper (dashed line) and lower (solid line) caustic for a limited range of $\varphi$ values. (b) Yield at the caustic: experiment (solid line), full-TDCIS (dashed line), 5 $\mathrm{p}_{\mathrm{z}}$-TDCIS (dotted line). (c) Enhancement factor: ratio of experimental and $5 \mathrm{p}_{\mathrm{z}}$-TDCIS yields (black line), ratio of full-TDCIS and $5 \mathrm{p}_{\mathrm{z}}$-TDCIS yields (blue line), ratio of full-PICS and $5 \mathrm{p}_{\mathrm{z}}$-PICS (red line). Dotted curves indicate a region where a single recombination event cannot be efficiently isolated. PICS taken from ref. [4].

In particular, we observe two caustics in correspondence of the green line on top of the figure, the dashed line indicating the higher energy caustic (upper caustic) and the solid line indicating the lower one (lower caustic). We show in Fig. 1(b) the harmonic yield along these two caustics (solid line). For comparing experimental data with theoretical results, we performed Time Dependent Configuration Interaction Singles (TDCIS) calculations. Dashed line in Fig. 1(b) shows TDCIS yields when correlations between all $5 \mathrm{p}, 5 \mathrm{~s}$ and $4 \mathrm{~d}$ orbitals are considered (full-TDCIS). Dotted line shows TDCIS yields when only the $5 \mathrm{p}_{z}$ orbital is interacting ( $5 \mathrm{p}_{\mathrm{z}}$-TDCIS). Figure 1(c) reports the theoretical enhancement factor (blue line), defined as the ratio between full-TDCIS and $5 \mathrm{p}_{\mathrm{z}}$-TDCIS yields, and the experimental enhancement factor (black line), which is given by the experimental yields normalized to the $5 \mathrm{p}_{\mathrm{z}}$-TDCIS yields. We observe good agreement between the experiment and full-TDCIS, which confirms that multi-electronic correlations with inner orbitals are enhancing the signal. The agreement is also good from a quantitative point of view apart from the region around $62 \mathrm{eV}$, where a single recombination event cannot be efficiently isolated. In this region the two curves are shown as dotted lines. 
In order to compare the observed results with another commonly used theoretical approach, we report as a red line in figure 1(c) the QRS enhancement factor calculated from ref. [3]. Within the QRS approach, the enhancement factor is the ratio between the partial photo-ionization cross section (PICS) of the target when all $5 \mathrm{p}, 5 \mathrm{~s}$ and $4 \mathrm{~d}$ orbitals are active (full-PICS) and the PICS when only the $5 \mathrm{p}_{z}$ orbital is active (5p $\mathrm{p}_{\mathrm{z}}$-PICS). It is clear from the comparison in fig. 1(c) that this procedure can not reproduce the experimental result. The need of ab-initio approach for the explanation of experimental observation is an indication that the multi-electronic resonance acts also on the recolliding electron wave-packet, as theoretically predicted in ref. [4].

These results trigger the question if this technique can be applied also to systems that do not show resonances. As a matter of fact, single atom theories predict a rapid fall of the signal for high energy caustics, as can be seen from Fig. 1(b). We report in Fig. 2(a) the experimental results obtained in argon, showing that the caustic can be detected even in the region of higher energies, due to the strong dipole response of argon beyond the Cooper minimum and in the absence of any resonance. A preliminary comparison with $3 p_{z}$-TDCIS harmonics reported in Fig. 2(b) shows that a good agreement is achieved even when only the external $3 p_{z}$ orbital is considered, as expected.


Fig 2. (a) HHG spectra in Ar as a function of $\varphi$. (b) 3pz-TDCIS HHG spectra as a function of $\varphi$. $(a, b)$ Green line indicates the position of the upper (dashed line) and lower (solid line) caustic.

\section{Conclusions}

In summary, we exploit caustics for performing detailed analysis on the xenon dipole response, reconstructing the enhancement factor given by the giant dipole resonance. This quantitative analysis was possible due to the interesting properties of caustics, where only one trajectory for each cycle of the laser field contributes to the HHG emission. Moreover, results in argon show that the technique can be easily extended to other targets even without the presence of resonances.

1. O. Raz, O. Pedatzur, B.D. Bruner, and N. Dudovich, Nat. Photon. 6(3), 170-173 (2012)

2. D. Faccialà, S. Pabst, B.D. Bruner, A.G. Ciriolo, S. De Silvestri, M. Devetta, M. Negro, H. Soifer, S. Stagira, N. Dudovich, and C. Vozzi, Phys. Rev. Lett. 117, 093902 (2016)

3. A.D. Shiner, B.E. Schmidt, C. Trallero-Herrero, H.J. Wörner, S. Patchkovskii, P.B. Corkum, J.C. Kieffer, F. Légaré, and D.M. Villeneuve, Nat. Phys. 7464 (2011)

4. S. Pabst and R. Santra, Phys. Rev. Lett. 111, 233005 (2013) 